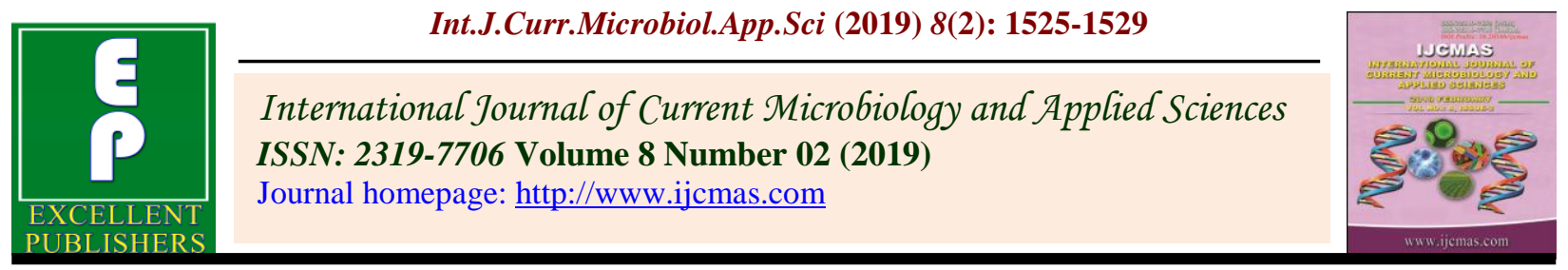

Original Research Article

https://doi.org/10.20546/ijcmas.2019.802.177

\title{
Preference of Internet on Other Media for Getting Information and Usage of Different Search Engines by Agricultural Research Scholars while using Internet of Agriculture Science in MPUAT, Udaipur (Rajasthan), India
}

\author{
L.R. Choudhary ${ }^{1 *}$, B.S. Bhimawat ${ }^{1}$ and Chamandeep Kaur ${ }^{2}$ \\ ${ }^{1}$ Department of Extension Education, Agriculture University, Jodhpur, (Raj.), India \\ ${ }^{2}$ Department of Home Science (Raj)., Agriculture University, Kota, India \\ *Corresponding author
}

\begin{abstract}
A B S T R A C T
Keywords

Agricultural

Research Scholars,

Internet of

Agriculture Science

Article Info

Accepted:

12 January 2019

Available Online:

10 February 2019

The present study was conducted at Rajasthan College of Agriculture, MPUAT, Udaipur. The RCA was selected because it is the oldest agriculture college in Rajasthan state and have adequate facilities of internet surfing for students, especially for research scholar at Central Library, departments and hostels as compared to other agriculture college in Rajasthan. Considering the importance of the internet utilization the present investigation was carried out on "Attitude and Utilization Pattern of Internet among the Research Scholars of Agriculture Science in MPUAT, Udaipur Rajasthan". The sample size was 117 respondents (90 male and 27 female). The Present study was conducted to analysis usage of different search engines and preference of internet on media for getting information by research scholars. This study include different category for search engines like Google, Yahoo, Lycos, Infoseek, etc.
\end{abstract}

\section{Introduction}

Internet in Education has been incredibly useful as it facilitates both information and communication. The Internet has increased the accessibility of education at all levels and has turned out to be a giant repository of knowledge. Students can access free video lectures online and refer to encyclopedias and study material in multimedia formats; teachers can make their lessons interesting by incorporating online-based projects in their study plans; and educational institutions can reach out to a wider audience by offering quality online courses. Internet also acts as a platform for retired teachers to reach out children in poor countries and educate them. It can improve the quality of education in many ways. It opens doorways to a wealth of information, knowledge and educational resources, increasing opportunities for learning in and beyond the classroom. Teachers use online materials to prepare lessons, and students to extend their range of learning. Internet is not only a great place to gather and store information but also allows its easy retrieval whenever desired. In fact, it has turned out to be better than libraries when 
it comes to gathering information and doing research work. Online encyclopedias and variety of other resources allow students to study any desired topic in much greater detail. Teachers too suggest students to refer to specific websites at home for topics being covered in class. MHRD and IGNOU have also developed Sakshat Portal on the lines of MIT OCW as a digital repository of eBooks, study material and eJournals for students. IGNOU's eGyankosh is another digital repository of learning material.

The $21^{\text {st }}$ century is witnessing a communication revolution with information processing and retrieving which are being reliably done at incredible speeds. The most striking invention in the field of education is the integration of Information and Communication Technology (ICT) in education. One of the most significant developments in this technology is the growth of the "Internet". The word Internet is derived from the words "global" and "network". The internet can be defined as network of networks and is the world's largest and most widely used network. According to Oxford dictionary "Internet is an international computer network connecting other networks and computers from companies, universities, NGOs etc." The internet is a useful tool for all in a technologically sophisticated world (Rehman et al., 2010). The Internet has reshaped the way we communicate, work, play and how we understand the world around us. With the rise of the Internet as a digital highway for messages and images, people now have a fast, convenient and reliable means to transmit and receive information. Internet use is spreading rapidly into daily life and has an impact in many areas including the higher education system. Internet heralded the development and implementation of new and innovative teaching strategies in higher education institutions. Educators who advocate technology integration in learning process believe that it will improve learning and prepare students to effectively participate in the $21^{\text {st }}$ century workplace. It is now widely used as a research tool for news, education, entertainment and informal web-based education.

\section{Materials and Methods}

The study was purposively conducted in Rajasthan College of Agriculture. Udaipur due to the reason that this College is the oldest Agricultural College in Rajasthan state and enjoys more and adequate facilities of internet surfing for agricultural students, especially for research scholars at central library as well as in their respective departments and Hostels as compared to other Agricultural Colleges in Rajasthan From the RCA, Udaipur, a list of all the PG and Ph.D. research scholars was prepared from the student section of the college. There was 150 $\mathrm{PG}$ and $110 \mathrm{Ph}$. D research scholars, i.e. a total of 260 research scholars registered in second semester during the session 2011-12. Since the whole population was not too big, hence, the whole population as such of 260 research scholars (150 PG and $110 \mathrm{Ph}$. D research scholars) was treated as the respondents for the purpose of the study. The total size of the sample taken was 117 respondents (90 male and 27 female).

\section{Results and Discussion}

\section{Use of different search engines}

The data in table 1 depicted that majority of the male (MS 2.99) and female (MS 2.78) agricultural research scholars used Google and accorded it first rank, about 98.89 per cent of the male agricultural research scholars used it mostly and only 1.11 per cent used it sometimes whereas 88.89 per of the female agricultural research scholars used Google mostly and only 11.11 per cent had never used it. In case of male agricultural scholars 
Rediff (MS 2.28) was ranked second followed by Gmail (MS 2.04), Orkut (MS 1.86), Yahoo (MS 1.71), India times (MS 1.21), MSN (MS 1.14), Live (MS 1.13), Info Seek (1.11), Khoj (MS 1.10), Netscape (MS 1.09), Bing (MS 1.07), Vibisimo (MS 1.06), Lycos (MS 1.02), Ask jeeves (MS 1.01) and Alta vista (MS 1.01) which were ranked third, fourth, fifth, sixth, seventh, eighth, ninth, tenth, eleventh, twelfth, thirteenth, fourteenth, fifteenth and sixteenth respectively.

Whereas in case of female agricultural research scholars Gmail (MS 2.44) was ranked second, followed by Yahoo (MS 2.08), Orkut (MS 1.81) and Rediff (MS 1.70) which were ranked third, fourth and fifth respectively. The search engines like India times, MSN, Live, Info seek, Khoj, Netscape, Bing, Vibisimo, Lycos, Ask jeeves and Alta vista were never been used by female agricultural research scholars. The value of rank order correlation $\left(r_{s}\right)$ was found to be 0.58 for which the calculated value of ' $t$ ' (2.76) was higher than its tabulated value at 5 per cent level of significance. Hence the null hypothesis $\left(\mathrm{H}_{0.2 .14}\right)$ was rejected and alternate hypothesis was accepted.

This leads to the conclusion that there is significant correlation between the male and female agricultural research scholars with regard to their usage of different search engines. This might be to the reason that male and female agricultural research scholars were learnt from each other and mostly browsed together in their respective Department and college library. These findings are in line with the findings of Mulimani and Gudimani (2008), Mishra et al., (2011).

Table.1 Preference of internet on other media for getting information by agricultural research scholars

$\mathbf{n}=\mathbf{1 1 7}$

\begin{tabular}{|c|c|c|c|c|c|c|c|c|c|c|c|c|c|}
\hline \multirow[t]{2}{*}{ S.No. } & \multirow[t]{2}{*}{$\begin{array}{c}\text { Media } \\
\text { Categories }\end{array}$} & \multicolumn{6}{|c|}{$\begin{array}{l}\text { Male agricultural research scholars } \\
\qquad(\mathrm{n}=90)\end{array}$} & \multicolumn{6}{|c|}{$\begin{array}{l}\text { Female agricultural research scholars } \\
\qquad(n=27)\end{array}$} \\
\hline & & $\begin{array}{c}\text { Most } \\
\text { preferred }\end{array}$ & Preferred & $\begin{array}{c}\text { Less } \\
\text { preferred }\end{array}$ & $\begin{array}{c}\text { Not } \\
\text { preferred }\end{array}$ & MS & Rank & $\begin{array}{l}\text { Mostly } \\
\text { preferrec }\end{array}$ & Preferred & $\begin{array}{c}\text { Less } \\
\text { preferrec }\end{array}$ & $\begin{array}{c}\text { Not } \\
\text { preferred }\end{array}$ & MS & Ranl \\
\hline 1 & Radio & $\begin{array}{c}27 \\
(30.00)\end{array}$ & $\begin{array}{c}36 \\
40.00)\end{array}$ & $\begin{array}{c}20 \\
(22.22)\end{array}$ & $\begin{array}{c}7 \\
(7.78)\end{array}$ & 2.92 & $\mathrm{~V}$ & $\begin{array}{c}4 \\
(14.81)\end{array}$ & $\begin{array}{c}12 \\
(44.44)\end{array}$ & $\begin{array}{c}6 \\
(22.22)\end{array}$ & $\begin{array}{c}5 \\
(18.52)\end{array}$ & 2.56 & $\mathrm{VI}$ \\
\hline 2 & Television & $\begin{array}{c}53 \\
(58.89)\end{array}$ & $\begin{array}{c}26 \\
(28.89)\end{array}$ & $\begin{array}{c}7 \\
(7.78)\end{array}$ & $\begin{array}{c}4 \\
(4.44)\end{array}$ & 3.42 & III & $\begin{array}{c}19 \\
(70.37)\end{array}$ & $\begin{array}{c}3 \\
(11.11)\end{array}$ & $\begin{array}{c}4 \\
(14.81)\end{array}$ & $\begin{array}{c}1 \\
(3.70)\end{array}$ & 3.48 & II \\
\hline 3 & & $\begin{array}{c}62 \\
(68.89)\end{array}$ & $\begin{array}{c}19 \\
(21.11)\end{array}$ & $\begin{array}{c}7 \\
(7.78)\end{array}$ & $\begin{array}{c}2 \\
(2.22)\end{array}$ & 3.57 & II & $\begin{array}{c}18 \\
(66.67)\end{array}$ & $\begin{array}{c}5 \\
(18.52)\end{array}$ & $\begin{array}{c}4 \\
(14.81)\end{array}$ & $\begin{array}{c}0 \\
(0.00)\end{array}$ & 3.52 & I \\
\hline 4 & Magazines & $\begin{array}{c}27 \\
(30.00)\end{array}$ & $\begin{array}{c}39 \\
(43.33)\end{array}$ & $\begin{array}{c}19 \\
(21.11)\end{array}$ & $\begin{array}{c}5 \\
(5.55)\end{array}$ & 2.64 & VII & $\begin{array}{c}4 \\
(14.81)\end{array}$ & $\begin{array}{c}16 \\
(59.26)\end{array}$ & $\begin{array}{c}4 \\
(14.81)\end{array}$ & $\begin{array}{c}3 \\
(11.11)\end{array}$ & 2.78 & $\mathrm{~V}$ \\
\hline 5 & Exhibition & $\begin{array}{c}4 \\
(4.44)\end{array}$ & $\begin{array}{c}28 \\
(31.11)\end{array}$ & $\begin{array}{c}36 \\
(40.00)\end{array}$ & $\begin{array}{c}22 \\
(24.44)\end{array}$ & 2.15 & $\mathrm{X}$ & $\begin{array}{c}0 \\
(0.00)\end{array}$ & $\begin{array}{c}7 \\
(25.92)\end{array}$ & $\begin{array}{c}12 \\
(44.44)\end{array}$ & $\begin{array}{c}8 \\
(29.63)\end{array}$ & 1.96 & VIII \\
\hline 6 & Posters/chart & $\begin{array}{c}9 \\
(10.00)\end{array}$ & $\begin{array}{c}28 \\
(31.11)\end{array}$ & $\begin{array}{c}32 \\
(35.56)\end{array}$ & $\begin{array}{c}21 \\
(23.33)\end{array}$ & 2.78 & VI & $\begin{array}{c}0 \\
(0.00)\end{array}$ & $\begin{array}{c}5 \\
(18.82)\end{array}$ & $\begin{array}{c}15 \\
(55.56)\end{array}$ & $\begin{array}{c}7 \\
(25.92)\end{array}$ & 1.92 & IX \\
\hline 7 & Kisan mela & $\begin{array}{c}21 \\
(23.33)\end{array}$ & $\begin{array}{c}27 \\
(30.00)\end{array}$ & $\begin{array}{c}25 \\
(27.78)\end{array}$ & $\begin{array}{c}17 \\
(18.89)\end{array}$ & 2.58 & IX & $\begin{array}{c}3 \\
(11.11)\end{array}$ & $\begin{array}{c}7 \\
(25.92)\end{array}$ & $\begin{array}{c}11 \\
(40.74)\end{array}$ & $\begin{array}{c}6 \\
(22.22)\end{array}$ & 2.26 & VII \\
\hline 8 & $\begin{array}{l}\text { Face to face } \\
\text { communicatior }\end{array}$ & $\begin{array}{c}37 \\
(41.11)\end{array}$ & $\begin{array}{c}27 \\
(30.00)\end{array}$ & $\begin{array}{c}12 \\
(13.33)\end{array}$ & $\begin{array}{c}14 \\
(15.56)\end{array}$ & 2.97 & IV & $\begin{array}{c}8 \\
(29.63)\end{array}$ & $\begin{array}{c}11 \\
(40.74)\end{array}$ & $\begin{array}{c}3 \\
(11.11)\end{array}$ & $\begin{array}{c}5 \\
(18.52)\end{array}$ & 2.81 & IV \\
\hline 9 & Internet & $\begin{array}{c}65 \\
(72.22)\end{array}$ & $\begin{array}{c}16 \\
(17.78)\end{array}$ & $\begin{array}{c}5 \\
(5.56)\end{array}$ & $\begin{array}{c}4 \\
(4.44)\end{array}$ & 3.58 & I & $\begin{array}{c}16 \\
(59.26)\end{array}$ & $\begin{array}{c}4 \\
(14.81)\end{array}$ & $\begin{array}{c}1 \\
(3.70)\end{array}$ & $\begin{array}{c}6 \\
(22.22)\end{array}$ & 3.11 & III \\
\hline 10 & Film show & $\begin{array}{c}27 \\
(30.00)\end{array}$ & $\begin{array}{c}22 \\
(24.44)\end{array}$ & $\begin{array}{c}22 \\
(24.44)\end{array}$ & $\begin{array}{c}19 \\
(21.11)\end{array}$ & 2.63 & VIII & $\begin{array}{c}1 \\
(3.70)\end{array}$ & $\begin{array}{c}8 \\
(29.63)\end{array}$ & $\begin{array}{c}4 \\
(14.81)\end{array}$ & $\begin{array}{c}14 \\
(51.85)\end{array}$ & 1.85 & $\mathrm{X}$ \\
\hline
\end{tabular}

Rank correlation coefficient $\left(\mathrm{r}_{\mathrm{s}}\right)=0.81 \quad \mathrm{t}=2.83 *$ (Significant at 0.05 level of probability)

Tabulated value of $t$ at 0.05 level of probability with 8 degrees of freedom $=2.31$ 
Table.2 Usage of different search engines by agricultural research scholars

\begin{tabular}{|c|c|c|c|c|c|c|c|c|c|c|c|}
\hline \multirow{3}{*}{$\begin{array}{l}S \\
N o\end{array}$} & & \multirow{2}{*}{\multicolumn{5}{|c|}{ Male agricultural research scholars (n=90) }} & \multirow{2}{*}{\multicolumn{5}{|c|}{ Female agricultural research scholars $(n=27)$}} \\
\hline & \multirow{2}{*}{ Category } & & & & & & & & & & \\
\hline & & Mostly & Sometimes & Never & MS & Rank & Mostly & Sometimes & Never & MS & Rank \\
\hline 1 & Google & $\begin{array}{c}89 \\
(98.89)\end{array}$ & $\begin{array}{c}1 \\
(1.11)\end{array}$ & $\begin{array}{c}0 \\
(0.00)\end{array}$ & 2.99 & I & $\begin{array}{c}24 \\
(88.89)\end{array}$ & $\begin{array}{c}0 \\
(0.00)\end{array}$ & $\begin{array}{c}3 \\
(11.11)\end{array}$ & 2.78 & I \\
\hline 2 & Yahoo & $\begin{array}{c}31 \\
(34.44)\end{array}$ & $\begin{array}{c}49 \\
(54.44)\end{array}$ & $\begin{array}{c}10 \\
(11.11)\end{array}$ & 1.71 & $\mathrm{~V}$ & $\begin{array}{c}9 \\
(33.33)\end{array}$ & $\begin{array}{c}11 \\
(40.74)\end{array}$ & $\begin{array}{c}7 \\
(25.93)\end{array}$ & 2.07 & III \\
\hline 3 & Lycos & $\begin{array}{c}2 \\
(2.22)\end{array}$ & $\begin{array}{c}0 \\
(0.00)\end{array}$ & $\begin{array}{c}88 \\
(97.78)\end{array}$ & 1.02 & XIV & $\begin{array}{c}0 \\
(0.00)\end{array}$ & $\begin{array}{c}0 \\
(0.00)\end{array}$ & $\begin{array}{c}27 \\
(100.00)\end{array}$ & 1.00 & VI \\
\hline 4 & Info seek & $\begin{array}{c}4 \\
(4.44)\end{array}$ & $\begin{array}{c}2 \\
(2.22)\end{array}$ & $\begin{array}{c}84 \\
(93.33)\end{array}$ & 1.11 & IX & $\begin{array}{c}0 \\
(0.00)\end{array}$ & $\begin{array}{c}0 \\
(0.00)\end{array}$ & $\begin{array}{c}27 \\
(100.00)\end{array}$ & 1.00 & VII \\
\hline 5 & Ask Jeeves & $\begin{array}{c}0 \\
(0.00)\end{array}$ & $\begin{array}{c}1 \\
(1.11)\end{array}$ & $\begin{array}{c}89 \\
(98.89)\end{array}$ & 1.01 & $\mathrm{XV}$ & $\begin{array}{c}0 \\
(0.00)\end{array}$ & $\begin{array}{c}0 \\
(0.00)\end{array}$ & $\begin{array}{c}27 \\
(100.00)\end{array}$ & 1.00 & VIII \\
\hline 6 & Alta vista & $\begin{array}{c}0 \\
(0.00)\end{array}$ & $\begin{array}{c}1 \\
(1.11)\end{array}$ & $\begin{array}{c}89 \\
(98.89)\end{array}$ & 1.01 & XVI & $\begin{array}{c}0 \\
(0.00)\end{array}$ & $\begin{array}{c}0 \\
(0.00)\end{array}$ & $\begin{array}{c}27 \\
(100.00)\end{array}$ & 1.00 & IX \\
\hline 7 & Khoj & $\begin{array}{c}0 \\
(0.00)\end{array}$ & $\begin{array}{c}9 \\
(10.00)\end{array}$ & $\begin{array}{c}81 \\
(90.00)\end{array}$ & 1.10 & $\mathrm{X}$ & $\begin{array}{c}0 \\
(0.00)\end{array}$ & $\begin{array}{c}0 \\
(0.00)\end{array}$ & $\begin{array}{c}27 \\
(100.00)\end{array}$ & 1.00 & $\mathrm{X}$ \\
\hline 8 & Rediff & $\begin{array}{c}17 \\
(18.89)\end{array}$ & $\begin{array}{c}42 \\
(46.67)\end{array}$ & $\begin{array}{c}31 \\
(34.44)\end{array}$ & 2.28 & II & $\begin{array}{c}4 \\
(14.81)\end{array}$ & $\begin{array}{c}11 \\
(40.74)\end{array}$ & $\begin{array}{c}12 \\
(44.44)\end{array}$ & 1.70 & V \\
\hline 9 & India times & $\begin{array}{c}4 \\
(4.44)\end{array}$ & $\begin{array}{c}11 \\
(12.22)\end{array}$ & $\begin{array}{c}75 \\
(83.33)\end{array}$ & 1.21 & VI & $\begin{array}{c}0 \\
(0.00)\end{array}$ & $\begin{array}{c}0 \\
(0.00)\end{array}$ & $\begin{array}{c}27 \\
(100.00)\end{array}$ & 1.00 & $\mathrm{XI}$ \\
\hline 10 & Vibisimo & $\begin{array}{c}1 \\
(1.11)\end{array}$ & $\begin{array}{c}4 \\
(4.44)\end{array}$ & $\begin{array}{c}85 \\
(94.44)\end{array}$ & 1.06 & XIII & $\begin{array}{c}0 \\
(0.00)\end{array}$ & $\begin{array}{c}0 \\
(0.00)\end{array}$ & $\begin{array}{c}27 \\
(100.00)\end{array}$ & 1.00 & XII \\
\hline 11 & Bing & $\begin{array}{c}0 \\
(0.00)\end{array}$ & $\begin{array}{c}6 \\
(6.66)\end{array}$ & $\begin{array}{c}84 \\
(93.33)\end{array}$ & 1.07 & XII & $\begin{array}{c}0 \\
(0.00)\end{array}$ & $\begin{array}{c}0 \\
(0.00)\end{array}$ & $\begin{array}{c}27 \\
(100.00)\end{array}$ & 1.00 & XIII \\
\hline 12 & Netscape & $\begin{array}{c}1 \\
(1.11)\end{array}$ & $\begin{array}{c}6 \\
(6.67)\end{array}$ & $\begin{array}{c}83 \\
(92.22)\end{array}$ & 1.09 & $\mathrm{XI}$ & $\begin{array}{c}0 \\
(0.00)\end{array}$ & $\begin{array}{c}0 \\
(0.00)\end{array}$ & $\begin{array}{c}27 \\
(100.00)\end{array}$ & 1.00 & XIV \\
\hline 13 & Gmail & $\begin{array}{c}27 \\
(30.00)\end{array}$ & $\begin{array}{c}40 \\
(44.44)\end{array}$ & $\begin{array}{c}23 \\
(25.56)\end{array}$ & 2.04 & III & $\begin{array}{c}15 \\
(55.56)\end{array}$ & $\begin{array}{c}9 \\
(33.33)\end{array}$ & $\begin{array}{c}3 \\
(11.11)\end{array}$ & 2.44 & II \\
\hline 14 & Orkut & $\begin{array}{c}15 \\
(16.67)\end{array}$ & $\begin{array}{c}47 \\
(52.22)\end{array}$ & $\begin{array}{c}28 \\
(31.11)\end{array}$ & 1.86 & IV & $\begin{array}{c}4 \\
(14.81)\end{array}$ & $\begin{array}{c}14 \\
(51.85)\end{array}$ & $\begin{array}{c}9 \\
(51.85)\end{array}$ & 1.81 & IV \\
\hline 15 & MSN & $\begin{array}{c}1 \\
(1.11)\end{array}$ & $\begin{array}{c}11 \\
(12.22)\end{array}$ & $\begin{array}{c}78 \\
(86.67)\end{array}$ & 1.14 & VII & $\begin{array}{c}0 \\
(0.00)\end{array}$ & $\begin{array}{c}0 \\
(0.00)\end{array}$ & $\begin{array}{c}27 \\
(100.00)\end{array}$ & 1.00 & $\mathrm{XV}$ \\
\hline 16 & Live & $\begin{array}{c}3 \\
(3.33)\end{array}$ & $\begin{array}{c}6 \\
(6.67)\end{array}$ & $\begin{array}{c}81 \\
(90.00)\end{array}$ & 1.13 & VIII & $\begin{array}{c}0 \\
90.00)\end{array}$ & $\begin{array}{c}0 \\
(0.00)\end{array}$ & $\begin{array}{c}27 \\
(100.00)\end{array}$ & 1.00 & XVI \\
\hline
\end{tabular}

Rank correlation coefficient $\left(r_{s}\right)=0.58 \quad t=2.76 *$ (significant at 0.05 level of probability)

Tabulated value of $t$ at 0.05 level of probability with 15 degrees of freedom $=2.13$

Preference of internet on other media for getting information by agricultural research scholars

The table 2 revealed that majority of the male agricultural research scholars preferred "internet" (MS 4.44) for getting information as compared to other media, as 72.22 per cent most preferred it, 17.78 per cent preferred it, 5.56 per cent less preferred it and only 4.44 per cent not preferred it, and ranked it first followed by "Newspaper" (MS 3.57), "Television" (MS 3.42), "Face to face communication" (MS 2.97), "Radio" (MS 2.92), "Posters/ Charts" (MS 2.78),
"Magazine" (MS 2.64), "Film show" ( MS 2.63), "Kisan mela" (MS 2.58) and "Exhibition" (MS 2.15) which were ranked second, third, fourth, fifth, sixth, seventh, eighth, ninth and tenth respectively. Whereas, majority of the female agricultural research scholars most preferred "Newspaper" (MS 3.52) for getting information as compared to other media as 66.67 per cent most preferred it, 18.52 per cent preferred it and 14.81per cent less preferred it and ranked it first. It was followed by "Television" (MS 3.48), "Internet" (MS 3.11), "Face to face communication" (MS 2.81), "Magazine" (MS 2.78), "Radio" (MS 2.56), "Kisan mela" (MS 
2.26), "Posters/Chart" (MS 1.92), "Film show" (MS 1.85) and "Exhibition" (MS 1.67) which were ranked second, third, fourth, fifth, sixth, seventh, eighth, ninth and tenth, respectively.

The value of rank order correlation $\left(\mathrm{r}_{\mathrm{s}}\right)$ was found to be 0.81 for which the calculated value of ' $t$ ' (2.83) was higher than its tabulated value at 5 per cent level of significance. Hence the null hypothesis $\left(\mathrm{H}_{02.21}\right)$ was rejected and alternate hypothesis was accepted. This leads to the conclusion that there is a significant correlation between the male and female agricultural research scholars with regard to their preference of internet on other media for getting information.

In conclusion, majority of the male and female agricultural research scholars had used Google search engine, used Gmail services for e-mail, were highly aware about e- mail services of internet, most preferred the websites to search information, browsed the required information from the internet through "typing the web address directly", most satisfied with the internet and rated it as a good source of information and partially satisfied with internet facility. Majority of male agricultural research scholars preferred college library for internet access, had spent Rs. 50 to 100 per month to use internet, used internet to collect information for class assignments and preferred the internet for getting information as compared to other media. Whereas female agricultural research scholars preferred mostly department for internet access, had used internet without any expenditure, used internet mostly to communicate with others, and preferred the newspaper for getting information as compared to other media.

\section{References}

Mulinami, M. N. and Gudimani, S. B. 2008. Usage of Internet by Students and Research Scholars of karnatak University Library: A Survey. International CALIBER-2008, University of Allahabad, Allahabad, February 28-29 and March12008@ INFLIBNET Centre, Allahabad.

Anunobi, C.V. 2006. Dynamics of internet usage: A case of students of the federal university of technology Owerri (FUTO) Nigeria. Educational research and reviews, 1:192-195

Garhwal, S. 2010. Internet utilization behavior of Agricultural students of Swami Keshwanand Rajasthan Agricultural University, Bikaner M.sc. (Ag) Thesis.

Kumar, R. and Kaur, A. 2006. Internet use by teachers and students in Engineering colleges of Punjab, Haryana and Himachal Pradesh States of India: An analysis" Electronic Journal of Academic and Special Librarianship vol.7:61-63

Mishra, O.P, Kumar, S, Dash, D.K and Kumar, R. 2011. Under Graduate Agriculture Students and Uses of the Internet: Case of Banaras Hindu University". JCS, vol 29 April 2011.

\section{How to cite this article:}

Choudhary, L.R., B.S. Bhimawat and Chamandeep Kaur. 2019. Preference of Internet on Other Media for Getting Information and Usage of Different Search Engines by Agricultural Research Scholars while using Internet of Agriculture Science in MPUAT, Udaipur (Rajasthan), India. Int.J.Curr.Microbiol.App.Sci. 8(02): 1525-1529.

doi: https://doi.org/10.20546/ijcmas.2019.802.177 